\title{
Linear mappings preserving the copositive cone
}

\author{
Yaroslav Shitov
}

\begin{abstract}
Let $\mathcal{S}_{n}$ be the set of all $n$-by- $n$ symmetric real matrices, and let $\mathcal{C}_{n}$ be the copositive cone, that is, the set of all matrices $a \in \mathcal{S}_{n}$ that fulfill the condition $u^{\top} a u \geqslant 0$ for all $n$-vectors $u$ with nonnegative entries. We prove that a linear mapping $\varphi: \mathcal{S}_{n} \rightarrow \mathcal{S}_{n}$ satisfies $\varphi\left(\mathcal{C}_{n}\right)=\mathcal{C}_{n}$ if and only if

$$
\varphi(x)=m^{\top} x m
$$
\end{abstract}

for a fixed monomial matrix $m$ with nonnegative entries.

Keywords: linear preserver, copositive matrices 2010 MSC: 15A86, 15B48

Our paper is a contribution to the study of linear preservers, which aims to describe linear operators on matrix algebras with respect to either a given property of matrices or according to a function defined on them. As suggested in modern literature [9], this study dates back to a 1897 paper [3] by Frobenius, who described linear operators $\varphi$ on $n \times n$ complex matrices that satisfy $\operatorname{det} \varphi(x)=\operatorname{det} x$ for all $x$. This study remains attractive for many researchers in linear algebra, as shown by the work discussed in the surveys [5, 8, 9] and their citation counts. This paper continues the investigation of [4] and applies this study to another well known concept of linear algebra - we work with preserver problems on the copositive cone, an object that is being studied since at least as early as the 60's [10]. Besides from being an attractive object to study in its own right [1], the copositive cone is relevant in different contexts of optimization [2] and combinatorics [6].

Email address: yaroslav-shitov@yandex.ru (Yaroslav Shitov) 


\section{Our result}

In this section and the rest of our note, we deal with real matrices and vectors. They are called nonnegative if all numbers they are formed of are nonnegative. The vectors are assumed to be written as columns, and we write $x \geqslant 0$ if a vector $x$ is nonnegative. An $n \times n$ matrix is called monomial if it has exactly one non-zero entry in every row and in every column. As said in the abstract, the notation $\mathcal{S}_{n}$ stands for the set of all $n$-by- $n$ symmetric real matrices, and $\mathcal{C}_{n}$ is the set of copositive matrices, which are those elements $a \in \mathcal{S}_{n}$ that satisfy the inequality $u^{\top} a u \geqslant 0$ for all nonnegative $n$-vectors $u$. A linear operator defined on $\mathcal{S}_{n}$ as $x \rightarrow m^{\top} x m$, for a fixed nonnegative monomial matrix $m$, is called a monomial congruence.

Theorem 1. A linear mapping $\varphi: \mathcal{S}_{n} \rightarrow \mathcal{S}_{n}$ satisfies $\varphi\left(\mathcal{C}_{n}\right)=\mathcal{C}_{n}$ if and only if $\varphi$ is a monomial congruence.

This result was conjectured in a recent paper [4] of Furtado, Johnson, Zhang, who checked its validity with $n=2$, and also for mappings of the form $x \rightarrow s^{\top} x s$ with a fixed, not necessarily monomial, matrix $s$. The 'if' part of Theorem 1 is trivial, so we concentrate on the 'only if' direction.

\section{Auxiliary results}

In the rest of our paper, the letter $\varphi$ stands for a mapping as in Theorem 1, that is, a linear operator on $\mathcal{S}_{n}$ that satisfies $\varphi\left(\mathcal{C}_{n}\right)=\mathcal{C}_{n}$. We work with the standard Euclidean topology on the space of all $n \times n$ real matrices, and we write $\partial \mathcal{C}_{n}$ to denote the boundary of $\mathcal{C}_{n}$ in $\mathcal{S}_{n}$. We list several known propositions and give brief sketches of their proofs for completeness.

Claim 2. (See [4, Theorem 3.1].) The mapping $\varphi$ is bijective on $\mathcal{S}_{n}$, and its inverse $\varphi^{-1}$ satisfies $\varphi^{-1}\left(\mathcal{C}_{n}\right)=\mathcal{C}_{n}$.

Proof. The first part follows because $\mathcal{C}_{n}$ spans $\mathcal{S}_{n}$ over $\mathbb{R}$, and the assertion with the inverse follows from $\varphi\left(\mathcal{C}_{n}\right)=\mathcal{C}_{n}$ by applying $\varphi^{-1}$ to both sides.

Claim 3. (See [4, Corollary 3.2].) We have $\varphi\left(\partial \mathcal{C}_{n}\right)=\partial \mathcal{C}_{n}$.

Proof. Follows because $\varphi$ is bijective and continuous.

Claim 4. (See [7, page 595, Remark].) If $a \in \partial \mathcal{C}_{n}$, then there is a non-zero vector $\xi \geqslant 0$ such that $\xi^{\top} a \xi=0$. 
Proof. Pick a vector that minimizes the value $x^{\top}$ ax over the compact set of all vectors $x=\left(x_{1}, \ldots, x_{n}\right)$ such that $x \geqslant 0$ and $x_{1}+\ldots+x_{n}=1$.

Claim 5. (See [11, Theorem 3.2].) If $a \in \mathcal{C}_{n}$ and $\xi^{\top} a \xi=0$ for an n-vector $\xi$ with all coordinates strictly positive, then a $\xi$ is a zero vector.

Proof. Since any vector $x$ that lies sufficiently close to $\xi$ is nonnegative, it has to satisfy $x^{\top} a x \geqslant 0$. This means that the quadratic form $A(x)=x^{\top} a x$ attains its local minimum at $\xi$, which shows that the gradient $\partial A / \partial x$ should be zero at $\xi$. It remains to note that this gradient equals $2 a x$.

\section{The proof of Theorem 1}

For a fixed index $t \in\{1, \ldots, n\}$, we define $\mathcal{A}^{t}$ as the set of all matrices in $\mathcal{S}_{n}$ with zeros at the $(t, t)$ position and positive numbers everywhere else.

Claim 6. We have $\mathcal{A}^{t} \subset \partial \mathcal{C}_{n}$ and $\varphi\left(\mathcal{A}^{t}\right) \subset \partial \mathcal{C}_{n}$.

Proof. The first inclusion follows because copositive matrices have nonnegative diagonal, and the second one is guaranteed by Claim 3 .

Claim 7. The image $\varphi\left(\mathcal{A}^{t}\right)$ spans a codimension-one subspace of $\mathcal{S}_{n}$.

Proof. Follows because $\varphi$ is bijective.

In what follows, we associate any copositive matrix $b$ with the kernel ker $b=\left\{x \geqslant 0: x^{\top} b x=0\right\}$ of the corresponding quadratic form.

Claim 8. We have ker $b=\operatorname{ker} c$ for all $b, c \in \varphi\left(\mathcal{A}^{t}\right)$.

Proof. Since $\varphi^{-1}(b), \varphi^{-1}(c)$ belong to $\mathcal{A}^{t}$, we can find $a \in \mathcal{A}^{t}$ and $\varepsilon>0$ satisfying $\varphi^{-1}(b)=\varepsilon \varphi^{-1}(c)+a$, which shows that $b=\varepsilon c+\varphi(a)$. Since $\varphi(a)$ is copositive, the latter equation shows that $\operatorname{ker} b \subseteq \operatorname{ker} c$.

Claim 9. There is a nonnegative vector $u^{t}$ with exactly one non-zero coordinate such that $\operatorname{ker} b=\left\{\lambda u^{t} \mid \lambda \in \mathbb{R}, \lambda \geqslant 0\right\}$ for all $b \in \varphi\left(\mathcal{A}^{t}\right)$.

Proof. We find a non-zero vector $u \in \operatorname{ker} b$ by Claim 4 , and we define $\sigma$ as the set of all non-zero indexes of $u$. Since the principal submatrix $b(\sigma \mid \sigma)$ is still copositive, we can apply Claim 5 and get $b(\sigma \mid \sigma) u^{\prime}=0$, where $u^{\prime}$ is the vector obtained from $u$ by removing its zero coordinates. According to Claim 8, the vector $u$ belongs to the kernel of any $c \in \varphi\left(\mathcal{A}^{t}\right)$, so the condition $c(\sigma \mid \sigma) u^{\prime}=0$ is satisfied by all such $c$. Since this condition is a union of $|\sigma|$ independent linear equations, we get $|\sigma| \leqslant 1$ from Claim 7 . The existence of two non-collinear vectors in ker $b$ contradicts to Claim 7 as well. 
In what follows, we write $e_{i j}$ to denote the $(i, j)$ matrix unit. We define $\mathcal{T}_{n}$ as the set of nonnegative matrices with zeros at the diagonal, which are exactly the set of trace-zero copositive matrices.

Claim 10. There is a monomial congruence $\mu$ such that the mapping $\psi=$ $\varphi \circ \mu$ satisfies $\psi\left(e_{t t}\right)=e_{t t}+b_{t}$ with $b_{t} \in \mathcal{T}_{n}$, for all indexes $t$.

Proof. Let $\pi(t)$ be the index of the non-zero coordinate of a vector $u^{t}$ as in Claim 9. If we had $\pi(s)=\pi(t)=j$ for some $s \neq t$, all the matrices in $\varphi\left(\mathcal{A}^{s}+\mathcal{A}^{t}\right)$ would have zeros at the $(j, j)$ position, which is a contradiction because the linear span of $\mathcal{A}^{s}+\mathcal{A}^{t}$ is full-dimensional. So we see that $\pi$ is an injective mapping, and as such it is a permutation of $\{1, \ldots, n\}$. For any $k \neq j$, we have $k=\pi(q)$ with $q \neq t$, so the $(k, k)$ entry of $\varphi\left(e_{t t}\right)$ should be zero because $\varphi\left(e_{t t}\right)$ belongs to the closure of $\varphi\left(\mathcal{A}^{q}\right)$. So we have $\varphi\left(e_{t t}\right)=\alpha_{t} e_{j j}+b_{t}$ with a real number $\alpha_{t}$ and a trace-zero matrix $b_{t}$, and then $b_{t} \in \mathcal{T}_{n}$ because $\varphi\left(e_{t t}\right)$ is copositive. Finally, since the set $\mathcal{T}_{n}$ lies in the closure of $\mathcal{A}^{1} \cap \ldots \cap \mathcal{A}^{n}$, we have $\varphi\left(\mathcal{T}_{n}\right) \subseteq \mathcal{T}_{n}$ and then $\varphi^{-1}\left(\mathcal{T}_{n}\right) \subseteq \mathcal{T}_{n}$. This shows that $\alpha_{t} \neq 0$ and gives a desired result up to a monomial congruence.

Claim 11. For $\psi$ as in Claim 10 , we have $\psi(x)=x$.

Proof. We get

$$
\psi^{-1}\left(e_{t t}+0.5 b_{t}\right)=\psi^{-1}\left(e_{t t}+b_{t}\right)-0.5 \psi^{-1}\left(b_{t}\right)=e_{t t}-0.5 \psi^{-1}\left(b_{t}\right),
$$

and since the resulting matrix is copositive, the preimage $\psi^{-1}\left(b_{t}\right)$ should be collinear to $e_{t t}$. Taking the $\psi$, we see that $b_{t}$ is collinear to $\psi\left(e_{t t}\right)=e_{t t}+b_{t}$, so we have $b_{t}=0$. This result means that $\psi\left(e_{t t}\right)=e_{t t}$.

Finally, we write $\beta_{i j}=\psi\left(e_{i j}+e_{j i}\right)$ for $i \neq j$. As noted in the proof of the previous claim, $\beta_{i j}$ is trace-zero. The matrix $\psi\left(e_{i i}+e_{j j}-e_{i j}-e_{j i}\right)=$ $e_{i i}+e_{j j}-\beta_{i j}$ is copositive, so the non-zero entries of $\beta_{i j}$ lie in the $2 \times 2$ submatrix with indexes $i, j$. This means that $\beta_{i j}=\alpha_{i j}\left(e_{i j}+e_{j i}\right)$ with some $\alpha_{i j} \in \mathbb{R}$, and we should have $\alpha_{i j} \leqslant 1$ again because $e_{i i}+e_{j j}-\beta_{i j}$ is copositive. The opposite inequality $\alpha_{i j} \geqslant 1$ follows by symmetry.

Claims 10 and 11 show that $\varphi$ is an identical mapping up to a monomial congruence, so the proof of Theorem 1 is complete. 


\section{References}

[1] A. Berman, M. Dür, N. Shaked-Monderer, Open problems in the theory of completely positive and copositive matrices, Electron. J. Linear Al. 29 (2015) 46-58.

[2] P. J. C. Dickinson, L. Gijben, On the computational complexity of membership problems for the completely positive cone and its dual, Comput. Optim. Appl. 57 (2014) 403-415.

[3] G. Frobenius, Über die Darstellung der endlichen Gruppen durch lineare Substitutionen, Sitzungsber. Deutsch. Akad. Wiss. Berlin (1897) 9941015 .

[4] S. Furtado, C. R. Johnson, Y. Zhang, Linear preservers of copositive matrices, Linear Multilinear A. (2019) doi.org/10.1080/03081087.2019.1692775.

[5] A. Guterman, C. K. Li, P. Šemrl, Some general techniques on linear preserver problems, Linear Algebra Appl. 315 (2000) 61-81.

[6] M. Hall Jr. Combinatorial Theory. Blaisdell, Lexington, 1967.

[7] J.-B. Hiriart-Urruty, A. Seeger, A variational approach to copositive matrices, SIAM Review 52 (2010) 593-629.

[8] C. K. Li, S. Pierce, Linear preserver problems, Am. Math. Mon. 108 (2001) 591-605.

[9] C. K. Li, N. K. Tsing, Linear preserver problems: A brief introduction and some special techniques, Linear Algebra Appl. 162 (1992) 217-235.

[10] J. E. Maxfield, H. Minc, On the matrix equation $X^{\prime} X=A, P$. Edinburgh Math. Soc. 13 (1962) 125-129.

[11] H. Väliaho, Criteria for copositive matrices, Linear Algebra Appl. 81 (1986) 19-34. 